\title{
Evaluación de la adaptación interna de resinas compuestas: Técnica incremental versus bulk-fill con activación sónica
}

\section{Internal adaptation evaluation of composite resins: Incremental filling versus Sonic-activated bulk-fill}

\author{
Pacheco Fernández, C*, Gehrkue Lorca, A*, Ruiz Araneda, P**, \\ Gainza Aragonés, P**
}

\begin{abstract}
RESUMEN
Introducción: Recientemente se han desarrollado las resinas bulk-fill que pueden ser utilizadas con técnica monoincremental, reemplazando la tradicional técnica incremental oblicua y acortando el tiempo clínico. El objetivo de este estudio es evaluar la adaptación de las resinas compuestas a las paredes internas cavitarias, utilizando técnica incremental oblicua y monoincremental con activación sónica.

Material y método: Se utilizaron 15 terceros molares sanos, a los cuales se les realizó dos preparaciones cavitarias clase II con márgenes en esmalte, de dimensiones en sentido mesiodistal de $3 \mathrm{~mm}$, vestibulolingual de $2,5 \mathrm{~mm}$, profundidad oclusopulpar de $2 \mathrm{~mm}$ y profundidad oclusoproximal de $4 \mathrm{~mm}$. Las preparaciones distales se restauraron utilizando la resina convencional Filtek ${ }^{\mathrm{TM}} \mathrm{Z} 350$ XT con técnica incremental y las mesiales con la resina bulk-fill SonicFill ${ }^{\mathrm{TM}}$ con técnica monoincremental y activación sónica. Las muestras fueron cortadas en sentido mesiodistal y analizadas mediante microscopio óptico a aumento de $10 \times$ y $40 \times$. Se midió el porcentaje de adaptación de ambas resinas y se realizó un test ANOVA de dos vías con prueba post hoc de Tukey.

Resultados: No se encontró diferencia estadísticamente significativa en la adaptación interna cavitaria utilizando la técnica monoincremental con activación sónica y la incremental oblicua ( $p>0,01)$.

Discusión: La técnica monoincremental con activación sónica, a pesar de tener un menor tiempo en el mercado, menor tiempo de entrenamiento clínico y menor consumo de tiempo clínico presentó valores similares de adaptación a las paredes internas cavitarias que la técnica incremental oblicua.
\end{abstract}

Palabras clave: Resinas Compuestas, Adaptación Dental Interna, Resina Bulk-fill, Técnica incremental.

\section{SUMMARY}

Introduction: Recently, Bulk-Fill resins have been developed and they can be used with a mono-incremental technique, eliminating the traditional oblique incremental technique and shortening the clinical time. The aim of this study is to evaluate the internal adaptation of composite resins using oblique incremental and monoincremental techniques with sonic activation.

Material and method: 15 healthy third molars were used and two enamel finished class II cavity was performed on each one of them. Dimensions were: $3 \mathrm{~mm}$ mesio-distal, $2.5 \mathrm{~mm}$ buccal-lingual, $2 \mathrm{~mm}$ occlusal-pulp depth, and $4 \mathrm{~mm}$ occlusal-proximal depth. The distal preparations were restored using a conventional Filtek ${ }^{\mathrm{TM}}$ Z350 XT (3M ESPE, USA) resin with oblique incremental technique and the mesial ones were restored using Bulk-Fill SonicFill $^{\mathrm{TM}}$ (Kerr, USA) resin through mono-incremental technique and sonic activation. The samples

* $\quad$ Escuela de Odontología, Universidad Austral de Chile, Valdivia, Chile.

** Profesor Adjunto, Biomateriales, Escuela de Odontología, Universidad Austral de Chile, Valdivia, Chile. 
were cut in a mesiodistal direction and analyzed with an optical microscopy at $10 \times$ and $40 \times$ magnification. The percentage of adaptation of both resins was measured, and a two-way ANOVA with Tukey correction was performed.

Results: No statistically significant difference was found in the adaptation of both resins, using oblique incremental and mono-incremental techniques with sonic activation $(p>0.01)$.

Discussion: Both techniques reported similar values of adaptation, therefore, the mono-incremental technique with sonic activation is a recently introduced technique, which seems to be less sensitive than oblique incremental technique.

Key words: Composite Resins, Dental Internal Adaptation, Bulk-fill resin, Incremental technique..

Fecha de recepción: 3 de julio de 2015.

Aceptado para publicación: 13 de julio de 2015.

Pacheco Fernández C, Gehrkue Lorca A, Ruiz Araneda P, Gainza Aragonés P. Evaluación de la adaptación interna de resinas compuestas: Técnica incremental versus bulk-fill con activación sónica. Av. Odontoestomatol 2015; 31 (5): 313-321.

\section{INTRODUCCIÓN}

Las resinas compuestas son el material restaurador de elección en la actualidad, presentando buenas propiedades mecánicas producto de la cantidad y naturaleza del relleno, capacidad de adhesión al diente mediante el uso de técnicas adhesivas, y otorgando la posibilidad de mimetizarse con la estructura dentaria, permitiendo buenos resultados estéticos $(1,2)$.

Un gran inconveniente de las resinas compuestas es su contracción de polimerización, la cual induce tensiones en la interfase diente-restauración, pudiendo generar sensibilidad post operatoria, tinciones marginales y desadaptación, con la consecuente colonización bacteriana y formación de caries secundaria (1,3-5).

Con el propósito de disminuir los efectos de la contracción de polimerización se han implementado diversas soluciones: modificaciones químicas, desarrollo de técnicas, materiales adhesivos y evolución de las lámparas de fotocurado $(4,5)$.

Dentro de las técnicas restauradoras de resinas compuestas en el sector posterior, se sugiere la utilización de la técnica incremental oblicua (4), sin embargo, los estudios no han determinado que ésta garantice un correcto sellado de los márgenes de la restauración, ni la eliminación completa de las brechas marginales $(3,4,6,7)$.

Recientemente han aparecido las resinas "bulk-fill" que se utilizan con técnica monoincremental o "en bloque", aplicando espesores de hasta 4-5 mm para realizar la restauración (8). Un ejemplo de estas resinas es el sistema SonicFill ${ }^{\mathrm{TM}}$ (Kerr, USA), un material que presenta alto contenido de relleno en peso $(83,5 \%)(9,10)$ y utiliza una pieza de mano que emite energía sónica, permitiendo disminuir la viscosidad del material, lo que aumentaría su adaptación a las paredes cavitarias (11).

El objetivo de este estudio es evaluar la adaptación de dos resinas compuestas a las paredes cavitarias internas de una cavidad clase II, una convencional empleando la técnica incremental oblicua versus una bulk-fill utilizando la técnica monoincremental con activación sónica.

La primera hipótesis nula es que no existen diferencias significativas en el porcentaje de adaptación de resinas compuestas a las paredes cavitarias internas entre las técnicas incremental oblicua y monoincremental con activación sónica.

La segunda hipótesis nula es que no existen diferencias significativas en la adaptación de la resina compuesta entre paredes homólogas de ambas técnicas 
ni dentro de las paredes cavitarias internas de las resinas realizadas con una misma técnica.

\section{MATERIAL Y MÉTODO}

Se realizó un estudio cuantitativo experimental in vitro siguiendo las indicaciones de la pauta CONSORT 2012 (12), en el laboratorio de la clínica de Odontología de la Universidad Austral de Chile, Valdivia, Chile.

Previo a la recolección de las muestras se solicitó la aprobación del comité de ética de la Facultad de Medicina de la Universidad Austral de Chile.

El tamaño muestral fue determinado con el software EPIDAT 4.1 con un poder estadístico del $80 \%$, un intervalo de confianza del $99 \%$ y en base a las asunciones recopiladas de un artículo similar (5). Este análisis arrojó un tamaño de la muestra de 6 por grupo, sin embargo, este fenómeno presenta una distribución normal, por ende, para poder utilizar una prueba paramétrica el tamaño total de la muestra se definió arbitrariamente en 15.

\section{Selección y preparación de las muestras}

Quince terceros molares sanos, donados por pacientes entre 18 y 25 años, bajo consentimiento informado, con un mínimo de $7 \mathrm{~mm}$ de longitud mesiodistal y $5 \mathrm{~mm}$ de ancho vestíbulo-palatino/lingual. Se excluyeron molares con restauraciones, fracturas y/o presencia de caries, lo cual fue determinado mediante observación directa a un aumento $4 \times$ con una lupa (Bio-Art, Brasil).

Las muestras fueron sumergidas en cloruro de sodio al 0,9\%, en un recipiente cerrado hasta su manipulación, y el recambio de la solución se realizó cada 7 días. Los dientes fueron sometidos a limpieza para remover restos de tejido periodontal, tejidos blandos y duros. Esto se realizó mediante la utilización de Jacquette número 1/2 (Hu-Friedy, USA) y Contra Ángulo modelo Pana-Max (NSK, Japón) con escobilla de profilaxis de nylon, utilizando agua con piedra pómez durante 1 minuto por superficie. Para facilitar su manipulación, las superficies radiculares fueron incluidas en acrílico rosado de autocurado (Marche, Chile).

Un mismo operador en cada diente realizó dos preparaciones cavitarias clase II, mesial y distal con sus márgenes en esmalte, las cuales fueron preparadas con fresas de alta velocidad ISO 806314109524 010 (MICRODONT, Brasil) e ISO 806314109524 018 (KG, Brasil) bajo abundante irrigación. Cada fresa se utilizó en un máximo de 5 preparaciones.

La configuración cavitaria (Figura 1), fue corroborada mediante una sonda periodontal milimetrada Carolina del Norte (Hu-Friedy, USA). Los ángulos internos de las preparaciones fueron redondeados y

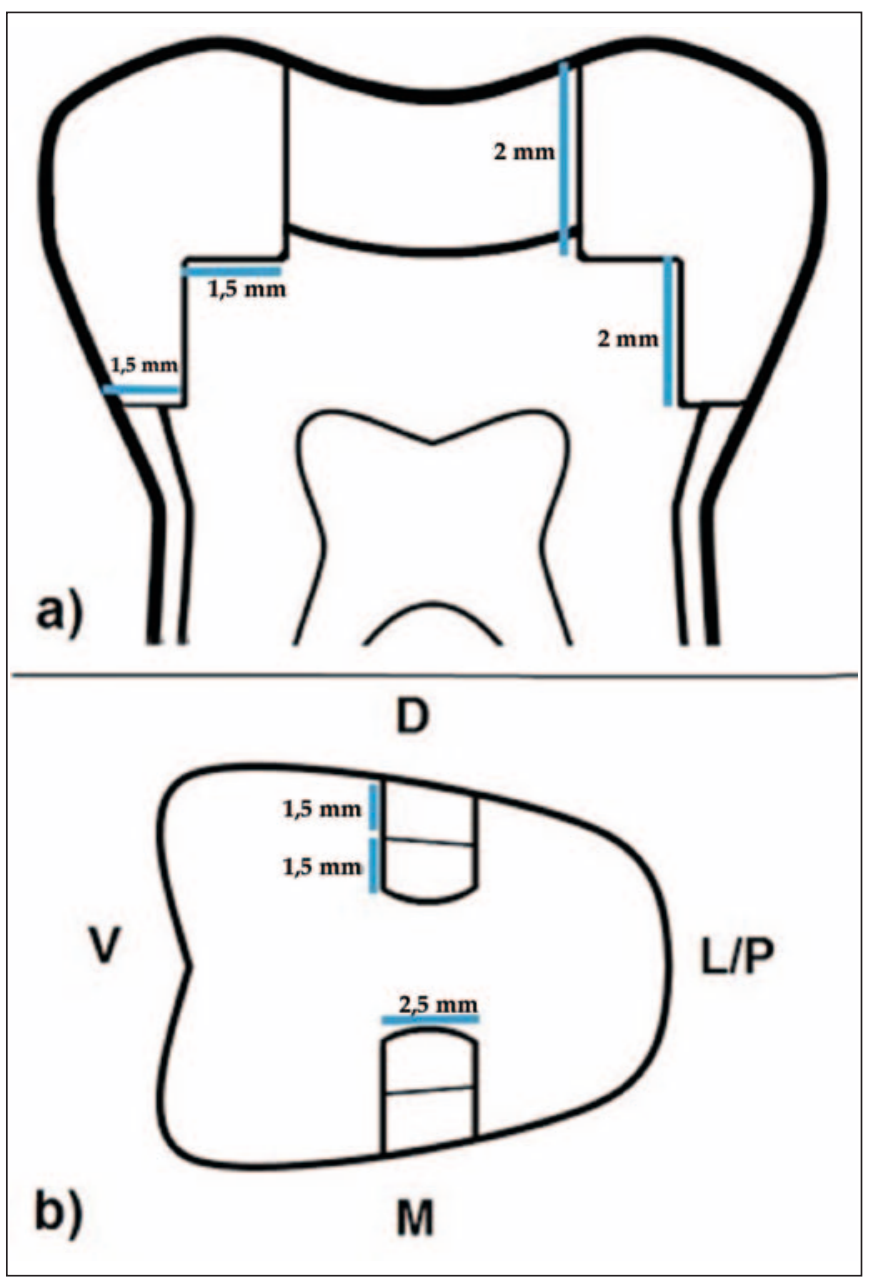

Fig. 1. Dimensiones de las preparaciones cavitarias. a) Vista longitudinal; b) Vista oclusal). V: Vestibular; L/P: Lingual/palatino; D: Distal; M: Mesial. 
fue corroborado por un mismo operador con control visual con lupa $4 \times$.

En todas las muestras se utilizó el sistema adhesivo Single Bond ${ }^{\circledR}$ Universal Adhesive (3M ESPE, Alemania) con técnica de grabado selectivo de esmalte, según instrucciones del fabricante.

A cada molar se le confeccionó una matriz de cobre individualizada, asegurada con un clamp metálico (Hu-Friedy, USA) y se verificó su adaptación a todas las paredes cavitarias.

La asignación del material restaurador a la preparación cavitaria fue definido al azar, ambas restauraciones realizadas por un mismo operador.

Las preparaciones mesiales se restauraron con resina compuesta SonicFill ${ }^{\mathrm{TM}}$ color A2 (Kerr, USA), utilizando la pieza de mano incluida en el sistema (Kerr, USA) en posición de intensidad 4, según instrucciones del fabricante con 1 incremento, y se fotoactivó con luz halógena modelo QHL75 (Dentsply, Alemania) por 40 segundos desde oclusal a $1 \mathrm{~mm}$ de distancia de la restauración. Las preparaciones distales se restauraron con resina compuesta convencional Filtek $^{\mathrm{TM}}$ Z350 XT, color A2 (3M ESPE, USA) con 5 incrementos de $2 \mathrm{~mm}$ y se fotoactivó cada uno de ellos por 20 segundos desde oclusal a $1 \mathrm{~mm}$ de distancia de la restauración.

Una vez retirada la matriz de cobre individualizada, se fotoactivó durante 20 segundos desde las superficie vestibulares y linguales/palatinos en ambas restauraciones.

La irradiancia de la lámpara halógena fue verificada con un radiómetro (SDI, Australia) cada 5 aplicaciones para constatar un mínimo de $580 \mathrm{~mW} / \mathrm{cm}^{2}$.

Los molares restaurados se sumergieron en cloruro de sodio al $0,9 \%$ por 96 horas y luego incluidos en bloques de acrílico de autocurado transparente (Veracril, Colombia). Posteriormente, se seccionaron en sentido mesiodistal con una cortadora de precisión seccional Isomet ${ }^{\mathrm{TM}}$ de baja velocidad (Buehler, China) con una hoja diamantada Isomet ${ }^{\mathrm{TM}}$ serie 15LC (Buehler, USA), a baja velocidad y con refrigeración constante, obteniendo cortes de 0,8 mm de grosor.

\section{Evaluación de los márgenes internos de las restauraciones}

Luego de cortadas, las muestras fueron analizadas y medidas con microscopio óptico, modelo CX41 (Olympus, Japón) a un aumento de $10 \times$ y $40 \times$ respectivamente.

Se consideró como adaptación interna del material restaurador a las paredes cavitarias, el margen regular sin pérdida de continuidad producto de burbujas y/o separaciones entre adhesivo y/o pieza dentaria y el material restaurador en cualquiera de sus paredes.

La adaptación del material restaurador a las paredes proximales, pulpares, axiales y gingivales (Figura 2) se cuantificó en micrones $(\mu \mathrm{m})$, utilizando el software computacional ImageJ. Estas mediciones se realizaron con la herramienta de mano alzada del software, con el fin de seguir fielmente el contorno

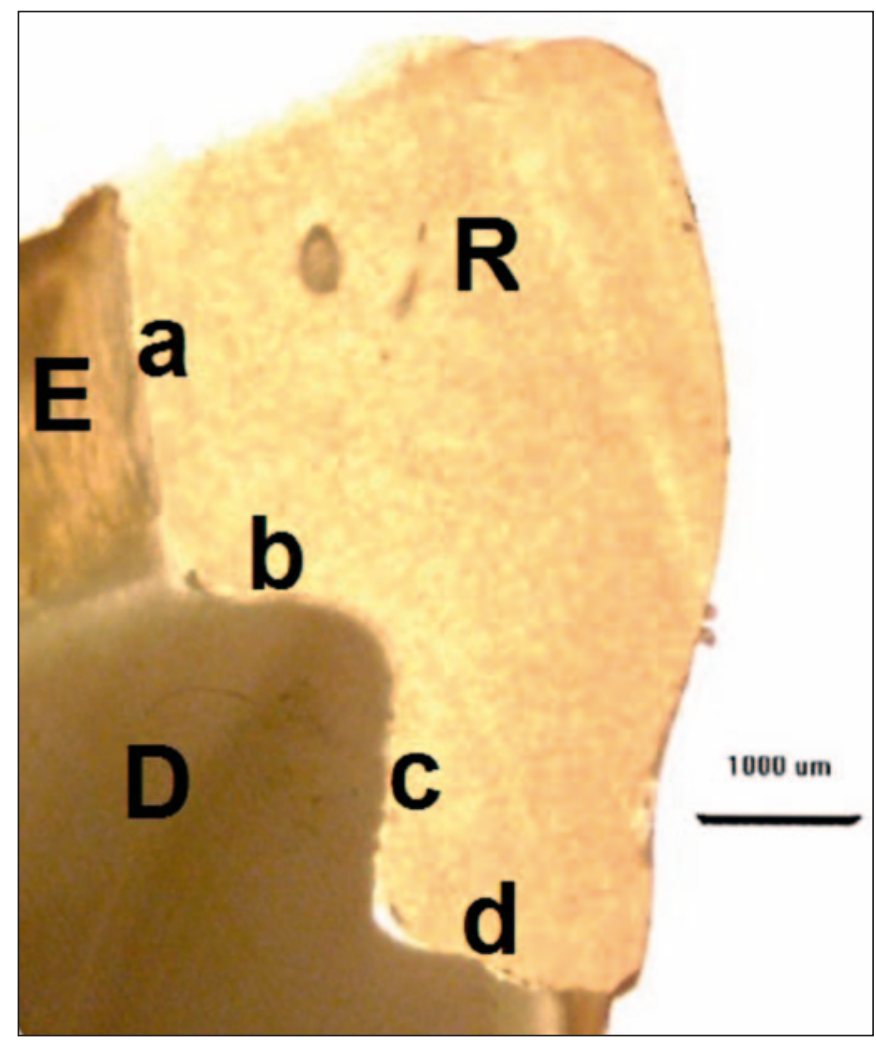

Fig. 2. Restauración de resina compuesta y descripción de las paredes cavitarias. R: Resina; E: Esmalte; D: Dentina; a: Pared proximal; b: Pared pulpar; c: Pared axial; d: Pared gingival. 
del margen. Los datos fueron tabulados y almacenados en hojas de cálculo de Google.

Se obtuvo la siguiente relación para determinar el porcentaje de adaptación a las paredes internas cavitarias: [suma de superficies adaptadas por pared (micrómetros)/longitud total de la pared (micrómetros) $\times 100]$.

\section{Método estadístico}

Para determinar la diferencia estadística en la adaptación interna cavitaria entre la técnica incremental oblicua y la monoincremental con activación sónica así como en la adaptación interna cavitaria en cada grupo de resina compuesta, fue utilizada la prueba paramétrica ANOVA factorial con prueba post hoc Tukey $(p<0,01)$ utilizando el software Graphpad PRISM 6.

\section{RESULTADOS}

Los porcentajes de adaptación total para la técnica monoincremental con activación sónica (SonicFill ${ }^{\mathrm{TM}}$ ) y para la técnica incremental oblicua (Filtek ${ }^{\mathrm{TM}} \mathrm{Z} 350$ XT) fueron de $82,01 \%$ y $79.85 \%$ respectivamente (Tabla 1). Los porcentajes de adaptación por pared se observan en la figura 3. Según los análisis empleados no existe diferencia estadísticamente significativa en la adaptación a las paredes cavitarias internas entre ambas resinas $(p<0,01)$. Por lo tanto, se acepta la primera hipótesis nula. Del mismo modo, no existen

\begin{tabular}{|c|c|c|}
\hline \multicolumn{3}{|c|}{$\begin{array}{c}\text { TABLA 1.- PORCENTAJE DE ADAPTACIÓN } \\
\text { INTERNA AL TOTAL DE PAREDES } \\
\text { CAVITARIAS APLICADAS CON TÉCNICA } \\
\text { MONOINCREMENTAL CON ACTIVACIÓN } \\
\text { SÓNICA Y TÉCNICA INCREMENTAL } \\
\text { OBLICUA }\end{array}$} \\
\hline & $\begin{array}{c}\text { Técnica } \\
\text { monoincremental } \\
\text { con activación } \\
\text { sónica (SonicFill) }\end{array}$ & $\begin{array}{c}\text { Técnica } \\
\text { incremental } \\
\text { oblicua } \\
\text { (Filtek Z350) }\end{array}$ \\
\hline Porcentaje \pm DS & $82,01 \% \pm 3,47$ & $79,85 \% \pm 3,79$ \\
\hline
\end{tabular}

DS: Desviación estándar.

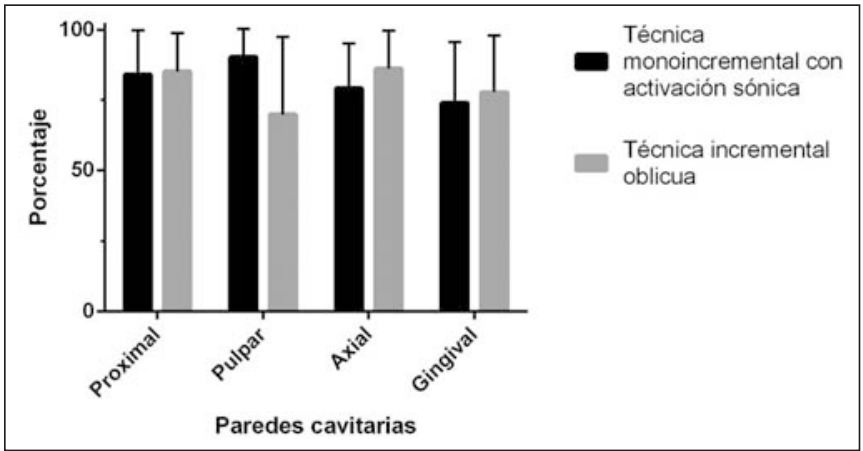

Fig. 3. Porcentajes de adaptación interna por pared cavitaria y técnica restauradora.

diferencias estadísticamente significativas en la adaptación de las resinas compuestas entre paredes cavitarias homólogas ni entre ellas dentro de un mismo material restaurador $(p>0,01)$ por lo que también se acepta la segunda hipótesis nula. La Figura 4 muestra la adaptación interna cavitaria de ambas técnicas. De la misma forma, la desadaptación a las paredes internas cavitarias de ambas técnicas se pueden observar en la Figura 5.

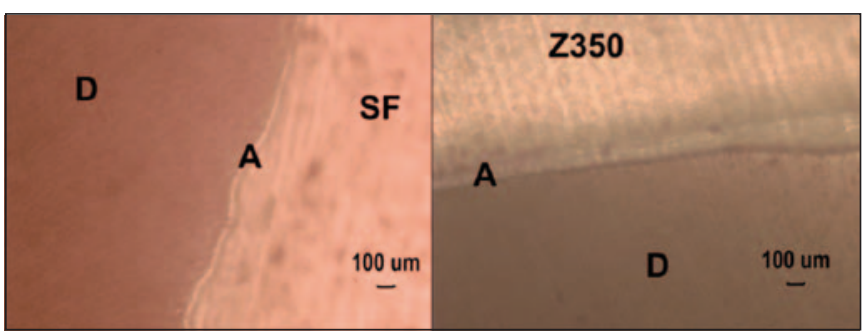

Fig. 4. Microscopía óptica 40×: Adaptación de ambas técnicas. D: Dentina; A: Adhesivo; SF: Técnica monoincremental con activación sónica (SonicFill); Z350: técnica incremental oblicua (Filtek Z350).

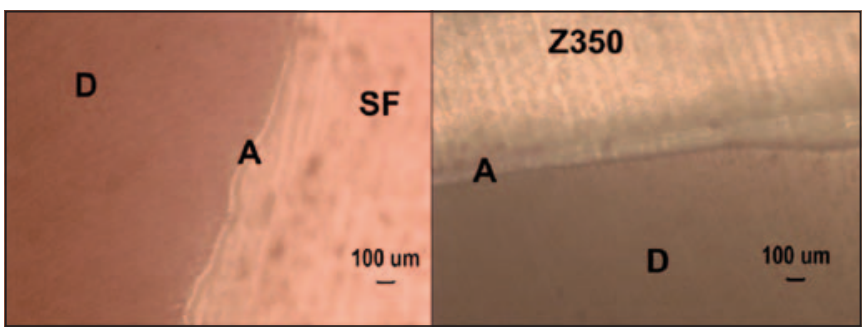

Fig. 5. Microscopía óptica 40×: Desadaptación de ambas técnicas. D: Dentina; A: Adhesivo; SF: Técnica monoincremental con activación sónica (SonicFill); Z350: técnica incremental oblicua (Filtek Z350); B: Burbuja. 


\section{DISCUSIÓN}

La técnica incremental oblicua, consigue disminuir el factor cavitario $(3,4,8,14)$, no obstante, esta técnica implica un mayor consumo de tiempo clínico (4). Por esta razón han aparecido las resinas compuestas bulk-fill que permiten la inserción del material restaurador en un sólo incremento $(14,15)$. Un representante de estas resinas es el sistema SonicFill ${ }^{\mathrm{TM}}$, que presenta una pieza de mano sónica de intensidad regulable, esto disminuye la viscosidad del material, aumentando la capacidad de adaptación a las paredes cavitarias (16).

Para aumentar la fluidez de una resina y con esto aumentar la adaptación, existen dos opciones: disminuir la cantidad de relleno o realizar una modificación reológica del material.

Campos y cols. compararon la adaptación marginal de cuatro resinas convencionales con sus versiones bulk-fill, no encontrando diferencias significativas entre los grupos (8). En su estudio evaluó la adaptación marginal de las resinas previamente y posteriormente a realizar carga y termociclado, los valores previos para SonicFill ${ }^{\mathrm{TM}}$ fueron similares a los de este estudio, levemente superior al $80 \%$, sin embargo, disminuyeron en un $17 \%$ después de la carga y termociclado. Una limitación del presente estudio es que no se realizó termociclado, ya que se deseaba aislar la variable técnica restauradora y adaptación a paredes internas cavitarias y no la estabilidad de la adaptación en el tiempo. Sería importante evaluar si el porcentaje de variación de la adaptación marginal posterior a la carga y termociclado es un factor predictor de la adaptación interna de la resina compuesta a las paredes cavitarias.

Dentro de los factores que intervienen en la adaptación de las resinas a las paredes cavitarias se encuentra el grado de viscosidad. Las resinas compuestas fluidas presentan mayor adaptación que las con alto contenido de relleno, sin embargo, presentan mayor contracción de polimerización y producen una mayor tensión en la interfase. No obstante, este efecto se atenúa debido a que poseen menor módulo de elasticidad, lo que implica una mayor deformación del material disminuyendo las tensiones en la interfase. Por otro lado, estas resinas presentan menor contenido de relleno lo que disminuye su resistencia mecánica, por lo que no debe ser expuesto a cargas masticatorias. Debido a esto, su indicación clínica se ve impedido como único material restaurador en el sector posterior $(2,17)$.

Tanto Uehara y cols. como Álvarez compararon la adaptación de SonicFill ${ }^{\mathrm{TM}}$ con resinas compuestas convencionales en preparaciones cavitarias clase I y II respectivamente $(5,17)$. Ambos obtuvieron resultados similares al presente estudio. La posible explicación de esta similitud es que todas las resinas estudiadas poseen alto contenido de relleno, tanto SonicFill ${ }^{\mathrm{TM}}$ (83,5\%), como Filtek ${ }^{\mathrm{TM}}$ Z350 (78,5\%) y Herculite ${ }^{\circledR}$ Précis $(78 \%)(4,13,18,19)$.

Por otro lado, Benetti y cols. midió la formación de brechas en resinas bulk-fill de baja y alta viscosidad utilizando técnica monoincremental, comparándola con una resina convencional con técnica incremental oblicua, y concluyó que la formación de brechas en las resinas bulk-fill con alto porcentaje de relleno fue similar a las resinas convencionales utilizando técnica incremental oblicua, al igual que el presente estudio. Y a la vez, las resinas bulk-fill con bajo contenido de relleno tuvieron resultados desfavorables en comparación a las anteriormente mencionadas (20).

Otro factor relevante es la profundidad de la preparación cavitaria, ya que, a medida que aumenta la profundidad se incrementa la posibilidad de desajuste y/o presencia de brechas (21). Esto se evidenció en el presente estudio, pues las paredes gingivales de las restauraciones con ambas técnicas presentaron valores bajos de adaptación (Figura 3). La posible explicación de esto es que al aumentar la profundidad de la preparación, disminuye la capacidad de control visual del operador.

Ortiz comparó la adaptación de una resina compuesta convencional fluidificada con un sistema que modifica su viscosidad (Compothixo, Kerr), y el sistema SonicFill ${ }^{\mathrm{TM}}$, encontrando una diferencia estadísticamente significativa en los resultados de ambas resinas, evidenciando un mayor desajuste en las convencionales (3). No obstante, sólo midió el margen cavosuperficial mediante un agente infiltrador y esto no permite evaluar el grado de adaptación total 
de una resina compuesta, por lo tanto, no es factible evaluar las posibles fallas que se pueden producir tanto en los márgenes internos como dentro del bloque restaurador. Un ejemplo de esto son las burbujas, que según nuestras observaciones se producen comúnmente en los ángulos internos. En ambas resinas se encontraron un total de 20 burbujas, correspondiendo 13 (65\%) a técnica monoincremental con activación sónica (SonicFill ${ }^{\mathrm{TM}}$ ) y 7 (35\%) a la técnica incremental oblicua (Filtek ${ }^{\mathrm{TM}}$ Z350 XT). En cuanto a la localización de éstas, en técnica monoincremental con activación sónica (SonicFill ${ }^{\mathrm{TM}}$ ), la mayor parte $(61,5 \%)$ se encontró en el ángulo axiogingival y en técnica incremental oblicua (Filtek ${ }^{\mathrm{TM}}$ Z350 XT) en el ángulo proximopulpar (57,1\%), y ya que el oxígeno presente en las burbujas es un inhibidor de la polimerización (21), se presenta un margen de resina sin polimerizar en la cual se pueden producir cambios dimensionales. Lo anterior está directamente asociado a la formación de brechas marginales (2), además de disminuir las propiedades mecánicas de la restauración y con esto su longevidad (22). En nuestro estudio se evaluó la capacidad de adaptación a las paredes internas, obteniendo resultados alrededor del $80 \%$ en ambas técnicas, lo que según Hickel y cols. es considerado como satisfactorio (11). Con respecto a la formación de brechas, es importante dilucidar si éstas son producto de la contracción de polimerización o son generadas por una adaptación deficiente durante la inserción de la resina en la preparación cavitaria.

La técnica incremental oblicua tiene una serie de desventajas (19). Es un procedimiento complejo, sensible, que demanda mayor tiempo clínico y requiere entrenamiento, ya que con facilidad se pueden generar vacíos o incorporar residuos entre los incrementos y/o en la interfase diente-restauración, lo que provoca una disminución de la adaptación de la resina $(3,4,24)$. Esto se evidencia en el grupo de la técnica incremental oblicua (Filtek ${ }^{\mathrm{TM}}$ Z350 XT) en las paredes gingival y pulpar, probablemente debido a un menor control visual del operador.

La adaptación en resinas compuestas depende de diversos factores relacionados a la preparación cavitaria: profundidad, extensión, complejidad y ubicación (20).
En el caso específico del sistema SonicFill ${ }^{\mathrm{TM}}$, podría desempeñar un rol importante la intensidad de la energía sónica de la pieza de mano, la cual posee cinco niveles, relacionados con la fluidificación de la resina lo que permite su adaptación a las paredes cavitarias. Por lo anteriormente mencionado, sugerimos realizar estudios que evalúen el efecto de la intensidad del sistema SonicFill ${ }^{T M}$ en el grado de adaptación.

Finalmente, se evidenció que a mayor profundidad se presentaron menores porcentajes de adaptación interna en ambas técnicas.

A pesar de las limitaciones de este estudio, se comparó una técnica que ha tenido una formación de pregrado establecida con protocolos y ampliamente difundida con una técnica recientemente introducida. Sin embargo, los valores alcanzados por ambas técnicas no presentan diferencias significativas entre sí, lo que evidencia el menor grado de sensibilidad de la técnica monoincremental con activación sónica.

\section{AGRADECIMIENTOS}

Esta investigación está basada en parte de los requerimientos de Camilo Sebastián Pacheco Fernández y Allyson Stephania Gehrkue Lorca para su titulación de Cirujano-Dentista en la Escuela de Odontología de la Universidad Austral de Chile.

Nuestros agradecimientos al Dr. Diego Halabí Robles, docente de la Escuela de Odontología, Universidad Austral de Chile, Chile; por su colaboración en este estudio.

Fuente de apoyo financiero: Este estudio fue realizado con el financiamiento de los autores, y con la ayuda de la Universidad Austral de Chile.

\section{BIBLIOGRAFÍA}

1. Hervás A, Martínez M, Cabanes J, Barjau A, Fos P. Resinas compuestas. Revisión de los materiales e indicaciones clínicas. Med Oral Patol Oral Cir Bucal 2006;11(2):15-20. 
2. Rodríguez D, Pereira N. Evolución y tendencias actuales en Resinas Compuestas. Act Odont Venez 2008;46(3):381-92.

3. Ortiz D, Masafierro M J. Adaptación, profundidad de polimerización y sellado marginal de restauraciones realizadas con una resina compuesta microhíbrida fluidificada y con Sonicfill ${ }^{\circledR}$. Rev Biomater Sociedad científica Grupo Chileno de Materiales Dentales 2014;1(2);32-53.

4. Botto I. Estudio Comparativo in vitro de la resistencia compresiva y la dureza superficial de un sistema de resina compuesta monoincremental (SonicFill $^{\mathrm{TM}}$ ) y uno convencional (Herculite ${ }^{\circledR}$ Precis)[citado 19 de Abril. 2015]. http://repositorio. uchile.cl/handle/2250/117406.

5. Uehara N, Ruiz AJ, Velasco J, Ceja I, Espinosa R. Adaptación marginal de las resinas bulk fill [citado 19 de Abril. 2015]. http://www.rodyb. com/adaptacion-marginal-de-las-resinas-bulkfill.

6. Liu F, Sun B, Jiang X, Aldeyab SS, Zhang Q, Zhu M. Mechanical properties of dental resin/composite containing urchin-like hydroxyapatite. Dent Mater 2014; 30(12):1358-68.

7. Ramírez RA, Setién VJ, Orellana NG, García C. Microfiltración en cavidades clase II restauradas con resinas compuestas de baja contracción. Act Odont Venez 2009;47(1):131-9.

8. Campos EA, Ardu S, Lefever D, Jassé FF, Bortolotto T, Krejci I. Marginal adaptation of class II cavities restored with bulk-fill composites. J Dent 2014;42(5):575-81.

9. Alshali RZ, Salim NA, Satterthwaite JD, Silikas N. Post-irradiation hardness development, chemical softening, and thermal stability of bulk-fill and conventional resin-composites. J Dent 2015;43 (2):209-18.

10. Garoushi S, Säilynoja E, Vallittu PK, Lassila L. Physical properties and depth of cure of a new short fiber reinforced composite. Dent Mater 2013;29(8):835-41.
11. Heintze SD, Monreal D, Peschke A. Marginal Quality of Class II Composite Restorations Placed in Bulk Compared to an Incremental Technique: Evaluation with SEM and Stereomicroscope. J Adhes Dent 2015;17(2):147-54.

12. Faggion CM. Guidelines for reporting pre-clinical in vitro studies on dental materials. J Evid Based Dent Pract 2012;12(4):182-9.

13. 3M ESPE. Perfil técnico del producto Filtek ${ }^{\mathrm{TM}}$ Z350 XT sistema restaurador universal 2010 [citado 23 de abril. 2015]. http://multimedia.3m. com/mws/media/7251770/perfil-tecnico-filtekz350-xt.pdf.

14. Kim RJ, Kim YJ, Choi NS, Lee IB. Polymerization shrinkage, modulus, and shrinkage stress related to tooth-restoration interfacial debonding in bulkfill composites. J Dent 2015;43(4):430-9.

15. Leprince JG, Palin WM, Vanacker J, Sabbagh J, Devaux J, Leloup G. Physico-mechanical characteristics of commercially available bulk-fill composites. J Dent 2014;42(8):993-1000.

16. Kerr Corporation. SonicFill ${ }^{\mathrm{TM}}$ Portfolio of Scientific Research 2011 [citado 23 de abril. 2015]. http:/ /www.sonicfill.es/media/4599/SonicFill_Portfolio. pdf.

17. Álvarez R. Descripción de la adaptación a tejidos dentarios del sistema de resina compuesta SonicFill (Kerr) y un material de restauración de resina compuesta convencional [citado 24 de abril. 2015]. http://www.repositorio.uchile.cl/ handle/2250/112719.

18. Alrahlah A, Silikas N, Watts DC. Post-cure depth of cure of bulk fill dental resin-composites. Dent Mater 2014;30(2):149-54.

19. Kerr Corporation. Catálogo Herculite Précis 2011 [citado 25 de abril. 2015]. http://intl.kerrdental. com/cms-filesystem-action?file=/kerrdental-pdf/ herculite_ss_spanish_web.pdf.

20. Benetti A, Havndrup-Pedersen C, Honoré D, Pedersen M, Pallesen U. Bulk-Fill Resin Compo- 
sites: Polymerization Contraction, Depth of Cure, and Gap Formation. Oper Dent 2015;40(2):190200.

21. Shawkat E, Shortall A, Addison O, Palin W. Oxygen inhibition and incremental layer bond strengths of resin composites. Dent Mater 2009;25(11):1338-46.

22. Caetano N, Grover V, López B, Henrique A. Influencia de la fotopolimerización gradual en el estrés de contracción en restauraciones de re- sina compuesta. Act Odont Venez 2008;46(3): 393-6.

\section{CORRESPONDENCIA}

Dr. Patricio Ruiz Araneda

Escuela de odontología. Facultad de medicina Universidad Austral de Chile

Rudloff 1640, Valdivia, Chile.

Email: pruizaraneda@gmail.com 